\title{
Maternally inherited sex ratio distortion as a result of a male-killing agent in Spilostethus hospes (Hemiptera: Lygaeidae)
}

\author{
FRANCIS R. GROETERS* \\ Molecular Evolution and Systematics Group, Research School of Biological Sciences, Australian National University, \\ PO Box 475, Canberra, A.C.T. 2601, Australia
}

\begin{abstract}
A family composed solely of daughters was produced by a pair of milkweed bugs, Spilostethus hospes (Hemiptera: Lygaeidae). The sex ratio among the offspring of the daughters was significantly female-biased, indicating that sex ratio distortion is heritable. The following results suggest that sex ratio distortion is caused by a maternally inherited, male-killing bacterium: females transmitted sex ratio distortion but males did not, egg hatch among pairs expressing sex ratio distortion was half that observed in pairs with unbiased offspring sex ratio, and pairs expressing sex ratio distortion converted to unbiased offspring sex ratio following tetracycline treatment. Successful selection for a highly female-biased sex ratio suggests that there is resistance to sex ratio distortion. Vertical transmission was incomplete and considerably reduced among females that underwent a forced delay in reproduction at cool temperatures analogous to an overwintering phase of the life cycle. An attempt to transfer bacteria horizontally by forcing early instars from a nonhost line to cannibalize host eggs was unsuccessful. With incomplete vertical transmission and no horizontal transmission, the bacterium presumably promotes its existence by boosting the reproductive success of host females, a possibility which remains to be investigated.
\end{abstract}

Keywords: horizontal transmission, male-killing bacteria, maternal inheritance, sex ratio distortion, Spilostethus hospes.

\section{Introduction}

Strong deviations from a 50:50 sex ratio in arthropods can be traced to a number of mechanisms. Obligate parthenogenesis (Lamb \& Willey, 1987), haplodiploidy (Wrensch \& Ebbert, 1992), meiotic drive (Lyttle, 1991), differential fertilization (Holloway, 1984; Hurst \& Vollrath, 1992), parthenogenesis arising from cytoplasmic incompatibility (Stouthamer et al., 1990; Zchori-Fein et al., 1992), feminization (Legrand et al., 1987), and early and late development male-killing (Hurst, 1991, 1993; Ebbert, 1992) have all been shown to cause highly distorted, usually female-biased sex ratios. Microorganisms are the causal agent in many cases of female-biased sex ratio. Because they are generally inherited through maternal cytoplasm, microorganisms have no evolutionary interest in being trans-

*Present address: Department of Biological Sciences, University of South Carolina, Columbia, SC 29208, U.S.A. mitted to males. They can reduce the chances of this by biasing the primary sex ratio toward females (Skinner, 1985; Hurst, 1992). The reason malekilling agents modify the secondary sex ratio is less clear, but clues have been provided by the timing of male death (Hurst, 1991; Hurst \& Majerus, 1992). In late development male-killing, death occurs at the fourth instar or later after a period of microbial proliferation. Male death serves to disperse microbial spores and promote horizontal transmission into other female lineages (Hurst, 1991). In early development male-killing, embryos are killed, so little time is available for microbial proliferation and horizontal transmission appears unlikely. Instead, embryonic death probably provides a resource advantage to sisters of dead males, either directly through consumption of inviable eggs or indirectly through reduced competition. This would boost reproductive success of both host females and the bacterial clonemates of the male-killers they harbour (Skinner, 1985; Hurst, 1991, 1993). Although 
evidence that female hosts can gain a fitness advantage through resource reallocation is sparse (Ebbert, 1992), the ecology during early development of many species appears conducive to such an effect (Hurst \& Majerus, 1992). Inbreeding and any associated inbreeding depression could also be reduced by male-killing (Werren, 1987; Ebbert, 1991), and would favour either early or late development malekilling.

Female-biased sex ratios caused by early malekilling agents, generally bacteria (Williamson \& Poulson, 1979; Gherna et al., 1991), are known from a wide variety of insect orders (Hurst, 1991, 1993; Ebbert, 1992). However, among the Hemiptera, only one case of distorted sex ratio as a result of malekilling has been reported, and although the agent of distortion was clearly maternally inherited, no attempt was made to test for the presence of a bacterium (Leslie, 1984).

In this study, I report on a maternally transmitted agent that causes sex ratio distortion by killing male embryos of the hemipteran Spilostethus hospes and provide suggestive evidence that the agent is a bacterium. Sex ratio distortion was suspected by discovery of an all-female family produced by a field-collected pair of bugs. Because infected females and their mates lose half their fitness through death of their sons, selection on nuclear genes should favour resistance to sex ratio distortion (Uyenoyama \& Feldman, 1978; Eberhard, 1980; Cosmides \& Tooby, 1981; Bull, 1983; Ebbert, 1992; Hurst, 1992). I tested for resistance by selecting for more strongly biased female sex ratios (and against resistance) in a distorted sex ratio line. If resistance exists or if for any other reason vertical transmission is less than 100 per cent, and there is little advantage gained by killing males through resource reallocation or inbreeding avoidance, then long-term persistence of a maternally inherited agent will depend on transmission between female lineages (Hurst \& Majerus, 1992). I tested for horizontal transmission by attempting to transfer sex ratio distortion into a line with no expression of the trait.

\section{Materials and methods}

\section{Collection site and husbandry}

A collection of 15 bugs was made from a patch of milkweed (Asclepias fruticosa) near Kingaroy in south-eastern Queensland, Australia in March 1992 (late summer). These bugs were used to establish a laboratory stock culture. The female that produced the all-female family was collected along with a single male from a nearby milkweed patch and kept separate from the main collection. Pairs were maintained in Petri dishes $(10 \mathrm{~cm}$ diameter $\times 2.5 \mathrm{~cm}$ deep) provided with sunflower seeds, a cotton-stoppered water vial and a cotton wad for oviposition. Offspring were reared in plastic cups provided with sunflower seeds and a water vial at a density of no more than 50 nymphs per cup. All bugs were kept in a temperature controlled room maintained at $25^{\circ} \mathrm{C}$.

\section{Selection experiment}

The 20 offspring from the all-female family were paired with males from the stock culture; 16 pairs produced offspring. Sex of offspring was assessed at the fifth (last) instar. Because sex ratio is a group trait it was necessary to employ family selection. The five pairs with the lowest percentage of male offspring were used to establish a 'low male' line, the five pairs with the highest percentage a 'high male' line, and offspring from the remaining pairs a control line on which no further selection was imposed. After the sex of each bug was determined, males and females were segregated. They remained segregated until the sex ratio of offspring from all pairs had been determined and selection could be applied. Three males and three females per family were used to set up pairs. Sib-sib matings were excluded. The three females from each family were mated with males from three different families, and likewise for males. There were occasions when not enough males were available from a selected family for use in setting up pairs. In these cases, more than three males from a selected family or males from other families within the line were used. From the total number of pairs that produced offspring (a total of 15 was always set up, and on no occasion did fewer than 10 pairs produce offspring), five pairs with extreme values for percentage male offspring were selected as parents of the next generation. In the control line, five pairs were chosen at random. For each pair, I set a goal to collect 150 eggs, but pairs were not excluded from the experiment unless they produced fewer than 25 eggs.

After three generations of selection, pairing of bugs to produce the fourth generation had to be delayed for 3 months, during which time bugs were maintained at $19^{\circ} \mathrm{C}$. Families were reduced in number and some completely lost during the delay; thus the number of pairs producing fourth generation offspring was greatly reduced compared with previous generations. At this point the high-male line was dropped owing to small numbers of generation 4 bugs. Also, selection on the low-male line was 
discontinued; rearing of the line was continued in order to provide material for other experiments. As a result of low egg production, the criterion for including a pair in the experiment was lowered to a minimum of 10 offspring. In addition to data on sex ratio, data were gathered on percentage egg hatch for each family, except for the families in generation 1.

\section{Maternal transmission}

Reciprocal crosses between the low-male and control lines were set up using generation 1 bugs to determine if sex ratio distortion was maternally transmitted. Four families from the control line and the four families with rankings of between six and nine from the low-male line provided adults. Three males and three females from each family were used to set up a total of 12 pairs for each type of reciprocal cross. Data were gathered on both sex ratio and percentage egg hatch.

\section{Tetracycline treatment}

Fourth generation adults from the low-male line were exposed to tetracycline to determine if the agent of sex ratio distortion was a bacterium. Thirty pairs of bugs were maintained on distilled water until they had produced at least 150 eggs. Distilled water was then replaced with a solution of 0.1 per cent $(0.1 \mathrm{~g} / 100 \mathrm{~mL})$ tetracycline $(50 \mathrm{~g} / \mathrm{kg}$ oxytetracycline hydrochloride) and subsequent egg clutches collected. Thirteen of the 30 pairs produced eggs both before and after tetracycline treatment. Sex ratio and percentage egg hatch were compared between the offspring of the two groups. Offspring from 11 of the 13 pairs were raised and used to set up 32 pairs of parents. Their offspring were used to determine if a 'curing' of sex ratio distortion among offspring of tetracycline-treated adults would be maintained in the subsequent generation. Eleven of the 32 pairs produced sufficient numbers of offspring for assessing sex ratio.

\section{Horizontal transmission}

Control line nymphs were fed eggs from the low-male line to determine if sex ratio distortion could be transmitted horizontally. Successful transmission would be indicated by sex ratio distortion among the offspring of treated bugs. A day before control line eggs were due to hatch, newly laid eggs from the low-male line were added to the rearing cup. No sunflower seeds were available to newly hatched nymphs; in order to survive they had to cannibalize eggs. After 5 days, nymphs were transferred to a new rearing cup provisioned with sunflower seeds and a water vial. This timing prevented contamination of control nymphs with newly hatched, low-male line nymphs because eggs take 7-8 days to hatch. Eighteen pairs of parents from generation 6 were set up and eight pairs produced offspring that were fed eggs and potentially could have obtained the sex ratio distortion trait. Twenty-four pairs of these generation 7 bugs were set up and 14 produced sufficient numbers of offspring to let sex ratio distortion be assessed.

\section{Results}

\section{Selection experiment}

The percentage of males among offspring of bugs in the all-female family was 36 per cent ( 261 males, 459 females). This is significantly different from a $50: 50$ sex ratio $\left(\chi_{1}^{2}=54.5, P<0.001\right)$. However, only 50 per cent of the 16 females produced female-biased progeny. This suggests that the trait can be spontaneously lost, or that there was resistance to sex ratio distortion among males from the stock culture. A single generation of selection produced significant differences between the control and low-male line for sex ratio [Fig. 1; Mann-Whitney-Wilcoxon test, (one-tailed): $U=138.5, P<0.03$ (one-tailed tests were employed because of the directional nature of the alternative hypotheses for a selection experiment)], but not between the control and high-male line $(U=129.5, P>0.15)$. A second generation of selection enhanced the difference between controls and the low-male line $(U=120, P<0.0001)$ and produced a significant difference between the highmale line and controls $(U=114.5, P<0.025)$. The level of sex ratio distortion reached a low of 11 per cent males in generation 3 (Fig. 1). The degree of distortion was even more pronounced than suggested by mean sex ratio in the line: in generation 3,50 per cent of all females failed to produce any male offspring and only one female out of 14 produced offspring with a 50:50 sex ratio. The response in the low-male line indicates that resistance to sex ratio distortion can be selected against, but even among low-resistance parents, the trait can still be lost in individual mothers. Selection on the high-male line did not cause a pronounced divergence in sex ratio compared with the control, but significant differences between this line and the control were maintained in generation $3(U=184.5$, $P<0.002)$. Differences between both the low- and 


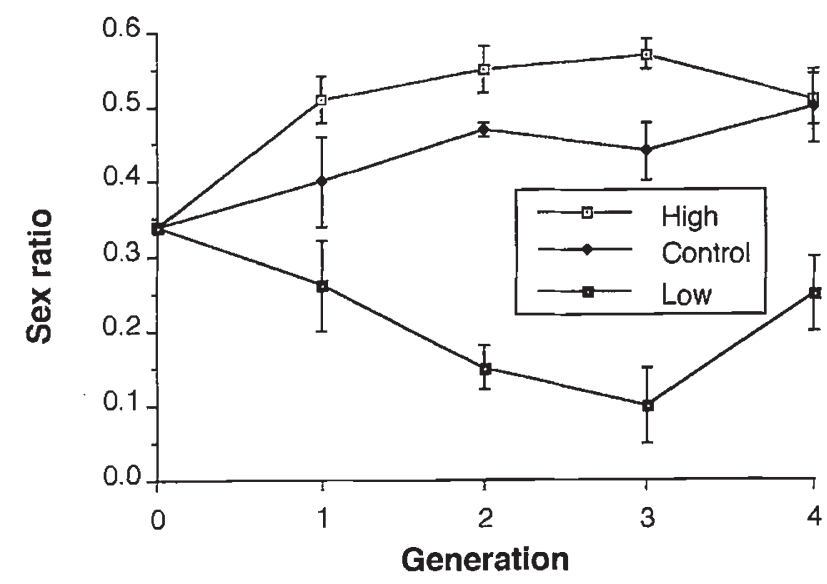

Fig. 1 Response to selection on sex ratio of Spilostethus hospes. Data are means of family values. Bars represent 1 $\mathrm{SE}$ of the mean.

high-male lines and the control were diminished in generation $4(U=28.5, P<0.02 ; U=8, P>0.5$, respectively), the generation that was produced from a small number of bugs following a 3-month delay in the experiment.

Percentage egg hatch in the low-male line was roughly half the hatch in either the control or highmale lines (Fig. 2). Differences between the low-male line and control were all significant $(U=119, \quad P<0.0001 ; \quad U=154, \quad P<0.005 ; \quad U=35$, $P<0.05$, for generations 2,3 and 4 , respectively). Percentage egg hatch of individual pairs was also strongly correlated with the sex ratio of their offspring (Spearman rank correlation $r_{\mathrm{s}}=0.62$, $P<0.0001)$. These results are all consistent with sex ratio distortion being the result of mortality of male embryos.

\section{Maternal transmission}

Sex ratio and egg hatch in crosses between low-male and control bugs varied according to female identity. Many pairs with low-male line females exhibited sex ratio distortion but no pairs with control females produced fewer than 43 per cent male offspring (Fig. 3). The mean sex ratio among offspring of pairs composed of low-male line females was $0.31 \pm 0.07$ compared with $0.54 \pm 0.02$ for pairs with control females. For egg hatch, the respective numbers were 45.5 per cent \pm 5.5 and 61.3 per cent \pm 7.7 . These differences are both significant (for sex ratio, $U=110.5, \quad P<0.025$; for egg hatch, $U=102.5$, $P<0.05)$. Clearly, males from the low-male line do not possess the capacity to transmit sex ratio distortion; this ability resides only with females. The

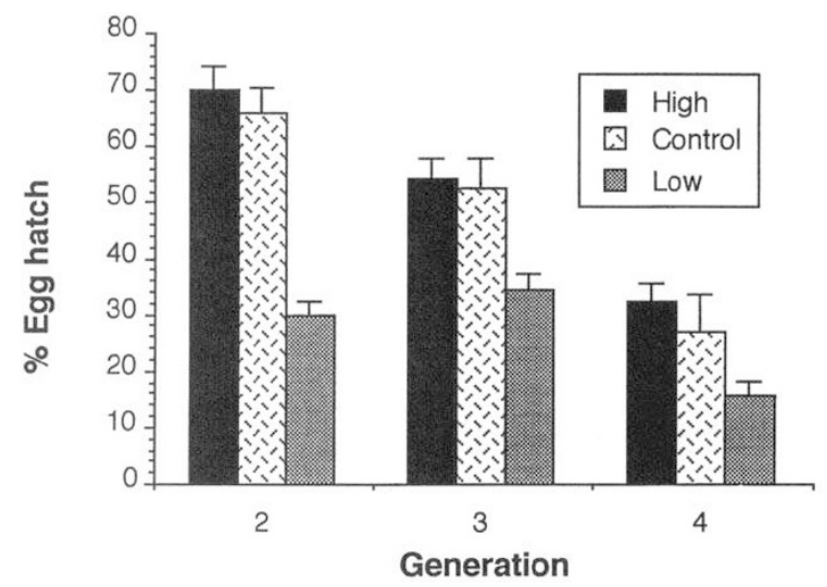

Fig. 2 Percentage egg hatch of Spilostethus hospes. Data are means of family values. Bars represent $1 \mathrm{SE}$ of the mean.

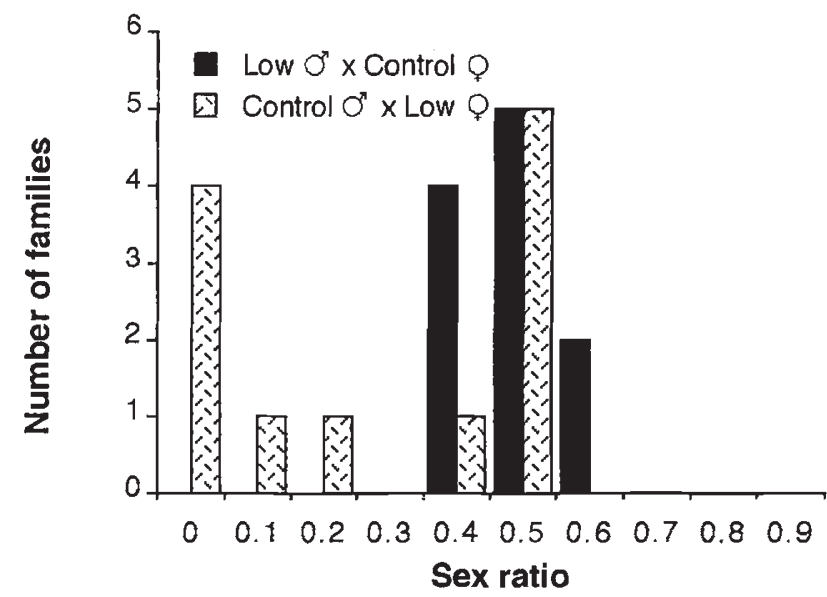

Fig. 3 Distribution of sex ratio in Spilostethus hospes for offspring of reciprocal crosses between low-male and control lines.

difference in egg hatch between the reciprocal crosses suggests that the difference in sex ratio is the result of higher male embryonic mortality.

\section{Tetracycline treatment}

The highly distorted sex ratios characteristic of the low-male line were converted to 50:50 sex ratios by treatment with tetracycline (Fig. 4). Before parents were treated, the average sex ratio among their offspring was $0.16 \pm 0.07$. After treatment these same pairs produced offspring with an average sex ratio of $0.57 \pm 0.03$. This difference is significant (Wilcoxon signed-rank test: $z=-2.83, P<0.0025$ ). Percentage egg hatch was also dramatically affected by tetracycline treatment. Percentage hatch was 24.3 


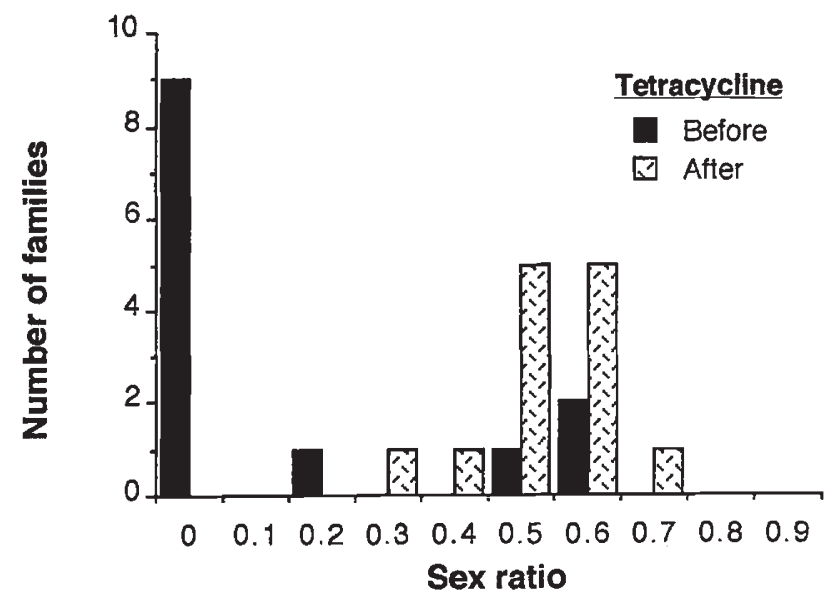

Fig. 4 Distribution of sex ratio in Spilostethus hospes for offspring produced before and after treatment of low-male line pairs with tetracycline.

per cent \pm 4.9 before treatment and 34.8 per cent \pm 3.8 after treatment $(z=-2.87, P<0.0025)$. The difference is even more pronounced if pairs not exhibiting sex ratio distortion before treatment are removed from the calculations. Percentage hatch is then 15.2 per cent \pm 1.9 before treatment and 29.3 per cent \pm 3.0 after treatment. This doubling following tetracycline treatment is precisely the magnitude expected if treatment restores normal sex ratios by preventing male embryos from being killed.

The 50:50 sex ratio among offspring of tetracycline-treated parents was maintained in the following generation, suggesting that these individuals had been 'cured' of the sex ratio distortion trait. The sex ratio among offspring of cured individuals was $0.52 \pm 0.05$, compared with $0.11 \pm 0.04$ among offspring of their uncured siblings (bugs produced before their parents were treated with tetracycline). One family (out of a total of 12) produced by cured parents did exhibit a significantly female-biased sex ratio (5 males, 35 females; $\chi_{1}^{2}=22.5, P<0.001$ ). This family was not followed further so it is unknown whether the biased sex ratio was a genuine expression of sex ratio distortion, but it suggests that curing with tetracycline can be incomplete.

\section{Horizontal transmission}

There was no evidence for distorted sex ratios among offspring of bugs fed eggs from the low-male line. Among 14 families the sex ratio was $0.55 \pm 0.03$. The most extreme female-biased sex ratio was 0.33 in a family of only 27 individuals; this sex ratio is not significantly different from 50:50 $\quad\left(\chi_{1}^{2}=3.0\right.$, $P=0.087)$.

(c) The Genetical Society of Great Britain, Heredity, 77, 201-208.

\section{Discussion}

Sex ratio distortion in $S$. hospes is caused by a cytoplasmically transmitted agent that kills male embryos. The female-biased sex ratio among offspring of daughters in the all-female family indicates that sex ratio distortion is heritable. However, only females from female-biased families were capable of transmitting sex ratio distortion; crosses with males from these families resulted in 50:50 sex ratios. This suggests that the trait is transmitted via maternal cytoplasm. A strong correlation between hatching success and sex ratio, and a percentage egg hatch among females expressing sex ratio distortion that was half the percentage among controls both indicate that sex ratio distortion occurs as a result of the death of male embryos. Tetracycline treatment of adults that were producing female-biased offspring rapidly resulted in the production of offspring with 50:50 sex ratios. These offspring appear to have been cured of the sex ratio distortion trait because they in turn produced offspring with normal sex ratios. Susceptibility of sex ratio distortion to tetracycline indicates that the agent of distortion is likely to be a bacterium that can be removed from a lineage or at least sufficiently reduced in number to enable male embryos to survive.

\section{Resistance}

Response to selection in the low-male line could have been the result of declines in genetically based resistance to bacteria, selection among bacteria for more efficient transmission or greater lethality, or a simple build-up of bacterial numbers over time. Bacterial titre can certainly play a role in transmission success. Build-up of bacterial numbers following treatment with antibiotics occasionally causes cured, sexually reproducing Trichogramma to return to parthenogenesis (Stouthamer et al., 1990). One female among offspring of tetracycline-treated bugs in this study also reverted to production of femalebiased offspring, and early male-killing in Drosophila is known to be sensitive to bacterial titre (Williamson \& Poulson, 1979). However, such an explanation for selection response in this study appears unlikely because the bacterial titre must have been high at the start of selection: it had to be sufficiently high to kill all male members of the all-female family used to found the selected lines. There is no reason to suspect that bacterial titre would have dropped in the next generation, then gradually increase over the next few generations. 
It seems more likely that the response was the result of selection against resistance to bacteria. The variance in offspring sex ratio among daughters in the all-female family suggests that there was variation for resistance to bacteria among stock parental males. Some males passed on genes that allowed their sons to survive exposure to male-killing bacteria, others did not. Alternatively, perhaps daughters were segregating for paternal genes that influence transmission rate. Other studies have shown that male identity can influence the degree of sex ratio distortion. In Oncopeltus fasciatus, crosses involving males from different geographical areas varied for sex ratio distortion (Leslie, 1984). There is abundant evidence for variation in both bacteria and host in the spiroplasma-Drosophila interaction (Williamson \& Poulson, 1979; Ebbert, 1991). Selection on sex ratio distortion caused by feminizing bacteria in an isopod also revealed nuclear genetic variation for resistance (Rigaud \& Juchault, 1992), and Holloway (1984) found variation for resistance to female-biased sex ratio in the rice weevil, Sitophilus oryzae. Selection for male-biased sex ratio in the high-male line in this study also provides some evidence for resistance, although the alternative explanation that male-killing bacteria were simply lost from the line because of incomplete vertical transmission cannot be excluded.

\section{Age effects}

A lengthy delay in the rearing of bugs diminished the expression of sex ratio distortion. The easing of sex ratio distortion could have been the result of low rearing temperature because declines in bacterial transmission rates are known to occur at both high and low temperatures (Ebbert, 1992, 1995). Alternatively, maternal age could have influenced transmission rate. Declines in transmission rate with age are known in other species with early male-killing (see Ebbert, 1992), and in other cases in which bacteria have effects on reproduction such as cytoplasmic incompatibility (Hoffmann et al., 1986). Leslie (1984) found no effect of maternal age on sex ratio distortion in $O$. fasciatus, but the comparison involved differences in age of 3 weeks, considerably less than the 3 month delay in this study. Because there was mortality during the rearing delay, some selection may also have occurred among individuals in the selected families. If prolonged proliferation of bacteria can have a detrimental effect on females, survivors of the rearing delay could have been those females with greater resistance to bacteria or simply females who, by chance, had lower bacterial titres.
They would be less likely to produce offspring with a biased sex ratio. Unfortunately, the effects of cool rearing temperature, maternal age, and withinlineage selection are confounded in this unplanned experiment. However, it certainly appears that transmission rate can be affected by a lengthy period of cool temperatures during which reproduction is suppressed. Transmission rates could vary over a life cycle and be lower across an overwintering period. Because precise estimates of transmission rate are needed to model the dynamics of maternally transmitted sex ratio distortion (e.g. Werren, 1987; Hurst, 1991), these results point to a need for examining transmission rates in the field over an entire life cycle, not simply under optimum culturing conditions in the laboratory.

\section{Persistence of sex ratio distortion}

One of the puzzles surrounding maternally inherited sex ratio distorters is their maintenance in populations given vertical transmission rates of less than 100 per cent and lack of evidence for horizontal transmission (Ebbert, 1992). Over time, the agent of distortion should disappear from the population. Yet in most species around 10 per cent of females harbour the distorting agent (Ebbert, 1991, 1992). Even though the frequency of host females in nature has not been investigated for $S$. hospes, the puzzle is probably applicable because vertical transmission rates were less than 100 per cent even in the line selected for lack of resistance, and no evidence for horizontal transmission was obtained in the experiment in which nymphs were fed eggs that presumably contained bacteria.

Somehow, female hosts must be gaining a reproductive advantage over nonhosts. Two potential mechanisms have been suggested (Hurst \& Majerus, 1992). One involves a reduction of inbreeding and any associated inbreeding depression (Werren, 1987). Nonhosts are considerably more likely to undergo inbreeding than hosts and so could suffer more from inbreeding depression than hosts. At present this possibility is impossible to test for $S$. hospes because no data are available on levels of inbreeding in nature nor on possible inbreeding depression. In this study, propagation of lines through the small number of individuals associated with a selection experiment appears to have had a detrimental effect on egg hatch (see Fig. 2). Thus, at least one fitness component appears to be susceptible to inbreeding depression. Little is known about the ecology of $S$. hospes, but its host plants include weedy species of the milkweed genus Asclepias 
(Thangavelu, 1978). Exploitation of these host plants typically favours a colonizing life history strategy (e.g. O. fasciatus; see Dingle, 1978), which, depending on the number of founders and their sources, and the number of generations produced before the next colonizing episode, could promote inbreeding. However, at present there are no data which would allow these parameters to be assessed for $S$. hospes.

A second mechanism by which female hosts may gain a reproductive advantage over nonhosts is through reallocation of resources from dead brothers to sisters (Skinner, 1985; Hurst, 1991). Perhaps early instar females gain a survival boost from consuming dead eggs. Egg cannibalism occurs in $O$. fasciatus (Dingle, 1968) and this behaviour was observed in S. hospes in this study. Although no data on fitness consequences of cannibalism are available, it seems likely that cannibalism could have a dramatic effect. As Hurst \& Majerus (1992) point out, first and second instar nymphs of $O$. fasciatus have poor survivorship (Sauer \& Feir, 1973), partly because of low searching capacity for seeds and difficulty in feeding from unopened seed pods (Ralph, 1977). The feeding ecology of $S$. hospes is likely to be similar to $O$. fasciatus because the species share host plant species, are both seedfeeders and are of similar size. Egg cannibalism may provide an alternative food source that can enhance survival through the critical early instars. Egg cannibalism may also boost the fitness of adult females. These possibilities remain to be investigated.

A second way that male death may facilitate reallocation of resources is through reduction of competition between brothers and sisters. The impact of intraspecific competition on individual fitness is unknown in $S$. hospes. However, as a colonizing species, resources are likely to be abundant compared with bug density. At collecting sites, $S$. hospes was easily outnumbered by an unidentified smaller hemipteran, suggesting that if competition is at all important, interspecific competition is likely to be more of a factor than intraspecific competition. Moreover, gregarious behaviour seems to be important for efficient feeding (Ralph, 1977) and probably for effective display of aposematic colouration to potential predators (Vulinec, 1990). Thus, the relationship of bug density to fitness will probably be a complicated function of a number of factors and some of these will have a positive effect on the relationship. Overall, reduced competition does not appear to be one of the more important means by which male death could boost the reproductive performance of female hosts in $S$. hospes or in general (Ebbert, 1992).
In summary, sex ratio distortion in $S$. hospes is the result of a maternally transmitted agent that acts by killing male embryos. For the first time in a hemipteran, there is evidence that sex ratio distortion is caused by a bacterium. Vertical transmission was found to be incomplete, even in a line in which resistance to sex ratio distortion was successfully selected against. This raises the issue of how the bacterium persists in populations. The possibility that persistence is based on horizontal transmission via egg cannibalism has been ruled out. Sisters of dead males probably obtain a reproductive advantage through avoidance of inbreeding depression or resource reallocation, but these possibilities remain to be investigated.

\section{Acknowledgements}

I thank M. Malipatil for providing species identification, H. Dingle and anonymous reviewers for comments on the manuscript, D. Shaw for providing laboratory facilities, and K. Strong for help in gathering data.

\section{References}

BULL, J. J. 1983. Evolution of Sex Determining Mechanisms. Benjamin/Cummings, Menlo Park, CA.

COSMIDES, L. M. AND TOOBY, J. 1981. Cytoplasmic inheritance and intragenomic conflict. J. Theor. Biol., 89, 83-129.

DINGLE, H. 1968. Life history and population consequences of density, photoperiod, and temperature in a migrant insect, the milkweed bug, Oncopeltus. Am. Nat., 102, 149-163.

DINGLE, H. 1978. Migration and diapause in tropical, temperate and island milkweed bugs. In: Dingle, $\mathrm{H}$ (ed.) The Evolution of Insect Migration and Diapause, pp. 254-276. Springer, New York.

EBBERT, M. A. 1991. The interaction phenotype in the Drosophila willistoni-spiroplasma symbiosis. Evolution, 45, 971-988.

EBBERT, M. A. 1992. Endosymbiotic sex ratio distorters in insects and mites. In: Wrensch, D. L. and Ebbert, M. A (eds) Evolution and Diversity of Sex Ratio in Haplodiploid Insects and Mites, pp. 150-191. Chapman and Hall, New York.

EBBERT, M. A. 1995. Variable effects of crowding on Drosophila hosts of male-lethal and non-male-lethal spiroplasmas in laboratory populations. Heredity, 74, 227-240.

EBERHARD, W. G. 1980. Evolutionary consequences of intracellular organelle competition. Q. Rev. Biol., 55, 231-249.

GHERNA, R. L., WERREN, J. H., WEISBURG, W., COTE, R., WOESE, C. R., MANDELCO, L. AND BRENNER, D. J. 1991. 
Arsenophus nasoniae gen.-nov., sp.-nov., the causative agent of the son killer trait in the parasitic wasp Nasonia vitripennis. Int. J. Syst. Bact., 41, 563-565.

HofFMANN, A. A., TURELl, M. AND Simmons, G. M. 1986. Unidirectional incompatibility between populations of Drosophila simulans. Evolution, 40, 692-701.

HOLloway, G. J. 1984. An analysis of inherited factors affecting the sex ratio in the rice weevil, Sitophilus oryzae L. Heredity, 55, 145-150.

HURST, G. D. D. AND MAJERUS, M. E. N. 1992. Why do maternally inherited microorganisms kill males? Heredity, 71, 81-95.

HURST, L. D. 1991. The incidences and evolution of cytoplasmic male killers. Proc. $R$. Soc. B, 244, 91-99.

HURST, L. D. 1992. Intragenomic conflict as an evolutionary force. Proc. $R$. Soc. B, 248, 135-140.

HURST, L. D. 1993. The incidences, mechanisms and evolution of cytoplasmic sex ratio distorters in animals. Biol. Rev., 68, 121-193.

HURST, L. D. AND VOLLRATH, F. 1992. Sex-ratio adjustment in solitary and social spiders. Trends Ecol. Evol., 7, 326-327.

LAMB, R. Y. AND WiLley, R. B. 1987. Cytological mechanisms of thelytokous parthenogenesis in insects. Genome, 29, 367-369.

LEGRAND, J. J., LEGRAND-HAMELIN, E. AND JUCHAULT, P. 1987. Sex determination in Crustacea. Biol. Rev., 62, 439-470.

LESLIE, J. F. 1984. A "sex-ratio" condition in Oncopeltus fasciatus.J. Hered., 75, 260-264.

Lyttle, T. w. 1991. Segregation distorters. Ann. Rev. Genet., 25, 511-557.

RALPH, C. P. 1977. Effect of host plant density on populations of a specialized, seed sucking bug, Oncopeltus fasciatus. Ecology, 58, 799-809.

RIGAUD, T. AND JUCHAULT, P. 1992. Genetic control of the vertical transmission of a cytoplasmic sex factor in Armadillidium vulgare (Latr.) (Crustacea, Oniscidea).
Heredity, 68, 47-52.

SAUER, D. AND FEIR, D. 1973. Studies on natural populations of Oncopeltus fasciatus (Dallas), the large milkweed bug. Am. Midl. Nat., 90, 13-37.

SKINNER, s. w. 1985. Son-killer: a third extrachromosomal factor affecting the sex ratio in the parasitoid wasp, Nasonia ( = Mormoniella) vitripennis. Genetics, 109, $745-759$.

STOUTHAMER, R., LUCK, R. F. AND HAMILTON, w. s. 1990. Antibiotics cause parthenogenetic Trichogramma (Hymenoptera/Trichogrammatidae) to revert to sex. Proc. Natl. Acad. Sci. U.S.A., 87, 2424-2427.

THANGavelu, K. 1978. Some notes on the ecology of three milkweed bugs in India (Heteroptera: Lygaeidae). $J$. Nat. Hist., 12, 641-647.

UYENOYAMA, M. K. AND FELDMAN, M. w. 1978. The genetics of sex ratio distortion by cytoplasmic infection under maternal and contagious transmission: an epidemiological study. Theor. Pop. Biol., 14, 471-497.

Vulinec, K. 1990. Aggregation by insects as a defense. In: Evans, D. L. and Schmidt, J. O (eds) Insect Defenses: Adaptive Mechanisms and Strategies of Prey and Predators, pp. 251-277. State University of New York Press, Albany, NY.

WERrEN, J. H. 1987. The coevolution of autosomal and cytoplasmic sex ratio factors. J. Theor. Biol., 124, 317-334.

W1Lliamson, D. L. AND POULSON, D. F. 1979. Sex ratio organisms (spiroplasmas) of Drosophila. In: Whitcomb, R. F. and Tully, J. G (eds) The Mycoplasmas, vol. 3, pp. 175-208. Academic Press, New York.

WRENSCH, D. L. AND EBBERT, M. A (eds) 1992. Evolution and Diversity of Sex Ratio in Haplodiploid Insects and Mites. Chapman and Hall, New York.

ZCHOR1-FEIN, E., ROUSH, R. T. AND HUNTER, M. S. 1992. Male production by antibiotic treatment in Encarsia formosa (Hymenoptera: Aphelinidae), an asexual species. Experientia, 48, 102-105. 